\title{
Difficultés rencontrées lors de la transmission d'exploitations agricoles. Le cas de la Bretagne
}

Problems encountered with farm transfers : the case of Brittany

\section{Romain Gaté et Laure Latruffe}

\section{(2) OpenEdition}

\section{Édition électronique}

URL : http://journals.openedition.org/economierurale/4792

DOI : $10.4000 /$ economierurale. 4792

ISSN : 2105-2581

\section{Éditeur}

Société Française d'Économie Rurale (SFER)

\section{Édition imprimée}

Date de publication : 15 février 2016

Pagination : 5-24

ISSN : 0013-0559

\section{Référence électronique}




\title{
Difficultés rencontrées lors de la transmission d'exploitations agricoles Le cas de la Bretagne
}

\author{
Romain GATÉ • CREM, UMR CNRS 6211, Université de Rennes 1 ; Agrocampus Ouest, UMR1302 \\ SMART, Rennes \\ romain.gate@univ-rennes1.fr
}

Laure LATRUFFE • INRA, UMR1302 SMART, Rennes; Agrocampus Ouest, UMR1302 SMART, Rennes laure.latruffe@rennes.inra.fr

En France, la transmission en agriculture est aujourd'hui un enjeu crucial puisqu'un chef d'exploitation sur deux était âgé de plus de 50 ans en 2010 et partira ainsi à la retraite au cours des dix prochaines années. L'objectif de cet article est de contribuer aux connaissances sur les enjeux et les difficultés liés au processus de transmission en agriculture, avec la Bretagne comme cas d'étude. Une revue de littérature, complétée par des entretiens auprès d'acteurs publics et professionnels intervenant dans le parcours de transmission et d'installation, a permis de mettre en lumière certains aspects clés, confirmés par une enquête exploratoire auprès de 15 agriculteurs installés depuis moins de cinq ans et de 25 agriculteurs pouvant partir à la retraite au cours des dix prochaines années en Bretagne.

MOTS-CLÉS : transmission, exploitation agricole, futur cédant, nouvel installé, stratégies d'investissement

\section{Problems encountered with farm transfers : the case of Brittany}

In France, farm transfers represent today a key strategic issue with half of all farm heads being over 50 years old in 2010 and therefore due to retire in the next ten years. The purpose of this paper is to contribute to our understanding of the issues and problems associated with the farm transfer process, taking the French region Brittany as a case study. A review of the literature rounded out by interviews with public and professional stakeholders involved in the transfer and settling processes, identified certain key aspects, which were borne out by an exploratory survey of 15 farmers established for less than five years and 25 farmers eligible for retirement in the next ten years in Brittany. (JEL: Q12)

KEYWORDS: transfer, farm, future transferor, newly settled farmer, investment strategies

D ans le cadre de la préparation de la Loi d'orientation agricole (LOA) de 2014, des assises régionales de l'installation se sont tenues de novembre 2012 à juillet 2013, au cours desquelles les enjeux et problématiques liés à la transmission ont été débattus ${ }^{1}$. La question de la continuité des exploitations agricoles s'est également posée récemment au Japon (voir par exemple Souma et Kiminami, 2011)

1. http://agriculture.gouv.fr/Assises-de-1-installation (consulté le 2 juillet 2014). et outre-Atlantique où les États-Unis ont mis en place, depuis le Farm Bill de 2008, un programme d'aide à l'installation en agriculture reposant sur des crédits et différents systèmes de garantie bancaire.

Plusieurs termes peuvent être recensés dans la littérature pour désigner une transmission: cession, vente, reprise, installation, succession, etc. Nous entendrons ici par transmission la passation d'une entreprise agricole dans sa totalité ou sa presque totalité, d'un cédant à un repreneur. Il s'agit ainsi d'au moins une 
installation pour un départ, contrairement aux démantèlements d'exploitation qui ne permettent qu'un agrandissement des exploitations existantes au détriment des installations. La transmission peut se réaliser dans un cadre familial ${ }^{2}$, avec une passation de l'exploitation entre générations, ou hors cadre familial avec la vente de l'exploitation à une personne extérieure à la famille. Plusieurs actifs matériels et immatériels peuvent faire l'objet de la transmission : le foncier en propriété et/ou en fermage, les bâtiments d'exploitation, la maison d'habitation, les machines agricoles, le cheptel, les droits à produire, les parts dans la société, les dettes, le savoir-faire, la gestion, la situation pédoclimatique et les risques inhérents à l'activité (Lobley et al., 2010 ; David, 1988). La transmission peut s'effectuer en une fois ou en plusieurs étapes ; elle peut également avoir fait l'objet d'une intégration progressive du successeur au sein de l'exploitation, par exemple comme associé ou aide familial. Dans le cas où le processus est progressif, l'agriculteur en place anticipe de préserver le patrimoine professionnel au sein de la famille par choix d'un fils comme futur successeur (Jacques-Jouvenot et Schepens, 2007). Le futur cédant transmet son savoir jusqu'à la transmission effective de son autorité sur l'exploitation. Lors de son installation, le repreneur peut continuer l'activité en place, introduire de nouvelles activités ou changer totalement l'orientation de production.

Les pouvoirs publics proposent aux agriculteurs qui s'installent la Dotation jeune agriculteur (DJA) $)^{3}$, allouée aux candidats à

2. En cadre familial, on considère les reprises d'exploitation derrière un membre de la famille jusqu'au troisième degré. Sur un arbre généalogique, il existe un degré entre un parent et son enfant, trois degrés de parenté entre un oncle et son neveu. En hors cadre familial, le repreneur n'a aucun lien de parenté avec le cédant.

3. Montant d'aide en trésorerie alloué par l'État selon différents critères fixés par chaque commission départementale d'orientation de l'agriculture (voir annexe 1). l'installation satisfaisant à certaines conditions, notamment d'âge (moins de 40 ans). Les acteurs privés proposent des accompagnements et/ou des conseils à la demande de leurs clients. Par exemple, une aide de 4000 euros est accordée aux exploitants qui s'inscrivent au Répertoire départs-installations (RDI). De plus, les conseillers transmission étudient la reprenabilité de l'exploitation et proposent différents scénarios de transmission.

La question des transmissions est aujourd'hui cruciale puisqu'un chef d'exploitation sur deux était âgé de plus de 50 ans en 2010 et partira ainsi à la retraite au cours des dix prochaines années (Gault et al., 2013a). Mais, comme mentionné plus haut, les départs à la retraite ne sont pas toujours suivis de nouvelles installations. En 2012, on dénombrait 12453 installations en France, soit $42 \%$ de moins qu'en 1997 (Pelc, 2014), et on comptait en Bretagne une installation d'un jeune de moins de 40 ans pour trois départs d'agriculteurs de plus de 50 ans (Chambres d'agriculture de Bretagne, 2014).

L'objectif de cet article est de contribuer aux connaissances sur les enjeux et les difficultés liés au processus de transmission en agriculture, avec la Bretagne comme cas d'étude, sur la base d'une revue de littérature, d'entretiens auprès d'experts et d'une enquête exploratoire auprès d'un échantillon non probabiliste d'exploitants volontaires des deux parties (exploitants devant céder leur exploitation dans les dix ans à venir et agriculteurs installés depuis moins de cinq ans). Le reste de l'article est organisé en trois parties. La deuxième partie décrit les enjeux et problématiques liés à la transmission relevés dans la littérature et recueillis auprès d'acteurs publics et professionnels du monde agricole impliqués dans le parcours des transmissions. La troisième partie présente l'enquête exploratoire réalisée auprès des agriculteurs, décrit la méthodologie de l'analyse statistique et en expose les principaux 
enseignements. La dernière partie discute les choix méthodologiques et conclut.

\section{Enjeux et problématiques liés à la transmission \\ Revue de littérature et opinions d'experts}

Une revue de littérature académique et grise a été combinée à des entretiens menés auprès d'experts. Ces entretiens semidirectifs, auprès de différents acteurs publics et professionnels du monde agricole (Crédit Agricole en Bretagne, ALTEOR Transaction, Chambre régionale d'agriculture de Bretagne, Société d'aménagement foncier et d'établissement rural [SAFER] Bretagne, Région Bretagne et Centre d'économie rurale [CER] d'Ille-etVilaine), impliqués directement ou indirectement dans les parcours de transmission des exploitations agricoles, ont été réalisés courant avril-mai 2014 en Bretagne.

\section{Préparation et anticipation}

Une difficulté majeure relevée dans la littérature et confirmée par les experts interrogés concerne le manque de préparation et d'anticipation de la transmission d'une exploitation du côté du cédant. En effet, une mauvaise préparation de la cession peut fragiliser la pérennité de l'exploitation et également la retraite du cédant. Selon les experts interrogés, la stratégie de transmission devrait être préparée cinq ans avant la date choisie du départ en retraite. Mais d'après La France Agricole (2014a), un horizon d'au moins dix ans est nécessaire.

\section{Le foncier : un enjeu majeur}

Le foncier est un enjeu majeur d'une transmission. Tout d'abord, un foncier très morcelé ne facilite pas la recherche d'un repreneur. En effet, le morcellement de la situation foncière de l'exploitation la rend moins attractive aux yeux des repreneurs, qui recherchent des exploitations avec un foncier bien structuré autour de bâtiments principaux, et non enclavé, si possible avec des terres à moins de 100 mètres de l'exploitation (La France Agricole, 2014b). De plus, une multitude de propriétaires fonciers est souvent synonyme de négociations complexes entre le cédant, les propriétaires et le repreneur. L'ensemble des propriétaires détenant les différents lots de l'exploitation en propriété doivent être d'accord pour que le bail soit poursuivi par le repreneur.

En plus de la difficulté liée à la multiplicité des propriétaires fonciers, une exploitation avec une part de fermage élevée dans sa Surface agricole utile (SAU) peut faire l'objet d'hésitation de la part de repreneurs potentiels. En effet, même si le statut du fermage en France limite (par exemple par des baux de long terme et par l'encadrement du loyer) les inconvénients liés au fermage pour l'exploitant locataire, celui-ci peut se sentir contraint dans ses décisions de production, d'investissement ou de pratiques. De plus, une partie non négligeable des subventions agricoles est capitalisée dans la valeur de la terre (voir par exemple Latruffe et Le Mouël, 2009), ce qui peut se traduire (de façon limitée) par une augmentation du montant du loyer demandé à l'agriculteur locataire.

D'un autre côté, une exploitation en propriété présente également un inconvénient : le prix des terres dans certaines régions peut freiner certains processus de transmission en augmentant le besoin de financement. Certains experts interrogés ont par exemple souligné des pressions foncières dans certaines zones, en témoigne le prix de la terre libre dans le pays de Fougères (dans le département d'Ille-et-Vilaine) qui atteint aujourd'hui 12000 euros par hectare selon les experts, soit plus de deux fois le prix moyen français. En effet, selon Safer-SSP-Terres d'Europe-ScafrINRA (2014), le prix moyen des terres et 
prés libres en Bretagne en 2013 était de 5240 euros par hectare. Les contraintes de financement du foncier peuvent ainsi être plus importantes dans ces zones pour les repreneurs.

\section{Freins économiques}

Le besoin de financement est une contrainte majeure aux transmissions. Selon l'Observatoire de l'installation et de la transmission en agriculture (Eoloas), le coût global d'une installation aidée (c'est-à-dire le coût de la reprise et le coût des investissements pendant les trois premières années suivant l'installation) est en moyenne de 285000 euros pour une exploitation individuelle et de 600000 euros pour une exploitation sociétaire (Exploitation agricole à responsabilité limitée [EARL] ou Groupement agricole d'exploitation en commun [GAEC]). Ce niveau de capitalisation peut dissuader les jeunes agriculteurs de reprendre une exploitation. Certaines filières sont plus touchées par ce problème, par exemple la filière porcine ou la filière marâichage où les exploitations ont une valeur des actifs très élevée.

Plusieurs valeurs peuvent être calculées pour une exploitation agricole (valeur patrimoniale, valeur de rendement ou de remboursement) et les différentiels entre ces valeurs peuvent varier du simple au triple selon Barthélémy (1997). Prenons le cas d'une exploitation ayant une valeur patrimoniale évaluée à 500000 euros et une valeur économique de seulement 300000 euros: les banques financeront 300000 euros et le repreneur devra apporter 200000 euros, ce qui représente un apport personnel conséquent. De plus, selon Gault et al. (2013a), la pratique du «pasde-porte » dans certaines régions et notamment dans le Nord de la France, pratique consistant à vendre des actifs à un prix supérieur à leur valeur de marché et pourtant interdite par le Code rural, entraîne une survalorisation du prix de cession.
Le coût des investissements pour moderniser l'exploitation et assurer sa viabilité, à la suite de l'installation, est un poids supplémentaire pour les repreneurs. Certains cédants préfèrent ainsi réaliser eux-mêmes les investissements nécessaires, avant leur retraite, afin de favoriser la transmission. En revanche, certains exploitants n'ayant aucun projet de transmission ni de successeur désigné désinvestissent dans leur outil de travail afin de vendre leurs biens à leur valeur de liquidation. L'outil ne valant plus rien, ces exploitations démantelées servent ensuite à agrandir les exploitations voisines (Calus et al., 2008 ; Boinon, 2011). Il existe aussi le cas où l'exploitant poursuit son activité au-delà de l'âge de la retraite sans anticiper une quelconque transmission, ce qui augmente les risques de mauvaise gestion et de liquidation des capitaux d'exploitation (Mishra et El-Osta, 2008).

De plus, le faible niveau de retraite anticipé par les cédants peut les inciter à prolonger leur activité agricole ou à garder tout ou une partie des terres en propriété, en vue de maintenir un revenu complémentaire à leur pension de retraite.

Enfin, on peut noter l'importance du contexte économique du secteur agricole sur les décisions de cession et de reprise. Les changements continuels de réglementations dans les attributions des aides de la Politique agricole commune (PAC), l'accumulation de contraintes environnementales et la volatilité des prix de plus en plus forte rendent l'avenir plus incertain, ce qui peut diminuer la volonté de transmettre de la part des cédants et de reprendre de la part des jeunes agriculteurs (Calus et al., 2008; Wheeler et al., 2012). Les experts interrogés ont par exemple souligné que les transmissions étaient difficiles dans le secteur des granivores, notamment dans un contexte de crise de la filière volaille de chair avec l'arrêt des subventions européennes pour le poulet export. Les repreneurs sont également peu nombreux pour 
la filière des bovins allaitants, qui souffre d'une faible rentabilité structurelle, ce qui peut être contraignant lors d'une reprise, le repreneur devant ainsi faire face à une période plus longue de remboursement des emprunts.

\section{Freins sociaux}

La recherche du repreneur ou du cédant «idéal » peut s'avérer complexe. Bien que la reproduction sociale soit forte dans le milieu agricole, des incertitudes demeurent. La logique patrimoniale amène à ce qu'un fils succède en priorité au père pour garantir la transmission intergénérationnelle du patrimoine. Pourtant, les agriculteurs n'assument pas le fait d'avoir à désigner un de leur(s) enfant(s) (JacquesJouvenot, 2012). Ils préfèrent laisser le doute subsister afin de ne pas prendre la responsabilité du choix de leur progéniture. Et selon Jacques-Jouvenot (2014a), par le partage des vies familiale et agricole, c'est finalement au successeur familial de s'autodésigner, mais cela impacte les stratégies à long terme des futurs cédants qui restent longtemps dans l'incertitude. Cette durée est confirmée par le dernier recensement agricole de 2010 qui indiquait que $52 \%$ des exploitants âgés de plus de 50 ans ne savaient pas à qui ils transmettraient leur exploitation et que $17 \%$ envisageaient la disparition de leur exploitation (Agreste, 2014). L'incertitude créée par cette problématique intrafamiliale peut menacer le développement et la transmissibilité de l'exploitation.

Il faut également prendre en compte les aspects psychologiques, certains cédants ayant également des difficultés à se séparer de leur outil de travail (Lobley et al., 2010). Deux cas de figure existent. Soit le cédant a un repreneur issu du cadre familial mais aimerait encore travailler longtemps sur l'exploitation, soit il n'a pas de successeur. Dans le premier cas, le cédant peut avoir des difficultés à partager son autorité décisionnelle avec son successeur, qui, lui, veut être autonome, ces conflits pouvant provoquer le départ de ce dernier (JacquesJouvenot, 1997 ; Levinson, 1971). Dans le second cas, poursuivre l'activité évite le sentiment d'échec lié à l'impossibilité de transmettre. Cela permet également d'atténuer le sentiment de culpabilité liée au fait d'être le dernier maillon qui n'a pas pu pérenniser le bien agricole à travers les générations (Bessière, 2008).

Céder son exploitation à une personne hors cadre familial peut être vécu comme un échec, comme souligné par Barthez et Charbonnier (2003), notamment parce que cela représente une rupture par rapport à la tradition familiale. Plusieurs raisons peuvent expliquer qu'aucun enfant ne succède aux parents. On peut mentionner une absence de fils, la présence d'une «fratrie » de filles sans gendre, ou encore des intégrations progressives qui échouent. De plus, même si le futur cédant a anticipé l'installation de son fils, l'arrivée d'une belle-fille ou encore des opportunités plus profitables dans un autre secteur économique peuvent inciter ce fils à renoncer à la reprise de ce «fardeau », comme suggéré par Gillet (1999). Enfin, il peut exister un décalage temporel entre la date de mise à la retraite et celle d'entrée dans la vie active des enfants du futur cédant. Le successeur familial pressenti pourra alors être différent de celui à qui le savoir-faire avait été transmis (souvent l'aîné), ce « mauvais » choix d'enfant pouvant mettre ce dernier en échec lors de son installation (Bessière, 2006). Dans ces deux cas, les futurs cédants doivent alors se tourner vers des personnes extérieures au cadre familial et qui représenteraient un profil idéal de repreneur en remplacement du fils qu'ils auraient aimé voir s'installer. Cependant, ils ne peuvent pas juger des compétences agricoles des successeurs hors cadre familial puisqu'ils ne les évaluent pas, d'où un risque de mettre en péril le devenir de l'exploitation. On peut également souligner, 
d'après nos entretiens auprès des experts et selon Gillet (1999), que plus la durée de négociation avec un tiers est longue, plus l'apparition de désaccords augmente.

Il s'agit de s'entendre non seulement sur le prix, mais également sur la date d'installation, sur la cession ou non de la maison d'habitation, sur le partage des rôles dans le cas de l'intégration d'un nouvel associé. Cette intégration peut par exemple révéler des dysfonctionnements existants au sein de la structure et créer des tensions entre les travailleurs présents (Barthez et Charbonnier, 2003). Ceci révèle l'importance qui doit être réservée à la définition d'une stratégie commune et d'un partage des tâches acceptés par l'ensemble des associés (La France Agricole, 2014b).

\section{Freins administratifs}

Enfin, parmi les difficultés souvent évoquées par les experts mais plus rarement mises en évidence dans la littérature concernant les transmissions d'exploitation agricole, on peut mentionner la complexité administrative de la démarche de transmission et le délai qui en découle. Ces tâches administratives ne font pas partie du cœur du métier de l'agriculteur. Elles sont considérées comme une surcharge en sus de l'ensemble des normes et règlements à respecter par ailleurs (Jacques-Jouvenot, 2014b).

Nos entretiens auprès des experts indiquent que les délais administratifs pouvaient représenter un frein à la transmission car, selon ces experts, il faut environ entre douze et dix-huit mois pour vendre l'outil de travail lorsque le repreneur réalise un parcours à l'installation avant de débuter une activité agricole, alors que les cédants aspirent à quitter leur métier rapidement une fois que leur décision est prise. Les projets de transmission sont soumis à un contrôle de la commission des structures qui vient s'ajouter aux autres contrôles de la Chambre d'agriculture et de la Commission départementale d'orientation agricole (CDOA) (Gault et al., 2013b). Les repreneurs bénéficiant d'aides publiques, quant à eux, doivent réaliser leur parcours à l'installation de huit mois. L'obtention de l'autorisation d'exploiter et le contrôle des structures prennent quatre à six mois environ. La SAFER est informée de tout acte de vente d'exploitation et de foncier agricole. Elle rend un avis dans les deux mois suivant la notification de la vente. D'autres délais administratifs pour les cédants sont liés au statut du fermage : il faut rechercher les propriétaires qui possèdent les terres pour obtenir l'agrément de poursuite du bail et présenter le nouvel exploitant. De plus, pour obtenir un accord bancaire, il faut compter deux à trois mois en sus.

À cela s'ajoute le délai pour trouver le repreneur ou le cédant «idéal » : selon les experts interrogés, les repreneurs inscrits au RDI qui recherchent des entreprises individuelles mettent environ deux années avant de trouver l'exploitation qui leur convient. De plus, selon Barthez et Charbonnier (2003), des conflits politiques peuvent retarder ou faire avorter une transmission. Certains syndicats se concurrencent dans des bassins de production et appuient chacun un candidat à l'installation sur une même exploitation. Par conséquent, cette concurrence peut menacer la négociation entre le cédant et le repreneur.

La littérature étant peu abondante sur les freins administratifs lors de la transmission des exploitations agricoles, nous nous sommes tournés vers la littérature sur l'entreprenariat en général. En ce qui concerne la transmission de Petites et moyennes entreprises (PME), la littérature grise et académique relève également la multiplicité des acteurs aux niveaux administratif et fiscal, ce qui complexifie cette étape cruciale pour l'entreprise (Verzele et Crijns, 2001). Cependant, ce sont les questions de performance, de montage financier et de 
relation cédant-repreneur qui sont surtout analysées et mises en exergue en général dans cette littérature (Benedsen et al., 2007 ; Cadieux et al., 2015).

\section{Enquête auprès $d$ 'un échantillon volontaire d'agriculteurs bretons}

\section{Description de l'enquête et de la méthodologie}

Un échantillon non probabiliste de 15 exploitants installés depuis moins de cinq ans (qu'on appellera par la suite « nouveaux installés ») et de 25 exploitants de plus de 50 ans qui n'avaient pas encore transmis leur outil de travail (qu'on appellera par la suite «futurs cédants ») ont été enquêtés en face-à-face en mai-juin 2014. L'enquête a couvert les quatre départements bretons. Les répondants n'ont pas été sélectionnés selon une procédure statistique de stratification dans l'ensemble des agriculteurs bretons : des contacts ont été obtenus par les experts mentionnés ci-dessus, et notamment ceux du Crédit Agricole. Seuls les agriculteurs volontaires et disponibles ont été enquêtés.

L'objectif de l'enquête exploratoire était d'identifier pour cet échantillon les principales difficultés rencontrées lors du processus de transmission et de tester nos hypothèses sur les difficultés rencontrées apportées par la littérature en les confrontant à la réalité du terrain. Le questionnaire comprenait des questions ouvertes et des questions fermées, et visait à collecter des informations qualitatives et quantitatives. Les informations qualitatives portaient notamment sur les difficultés rencontrées lors du parcours de transmission. Une liste de difficultés potentielles, sélectionnées sur la base de la revue de littérature et des entretiens avec les acteurs professionnels, était proposée aux répondants, qui choisissaient celles qu'ils avaient rencontrées. Ils devaient également choisir dans cette liste prédéfinie la difficulté la plus importante. Les cédants étaient également invités à décrire leur comportement d'investissement avant transmission. Concernant les informations quantitatives, elles portaient sur les caractéristiques structurelles et financières de l'exploitation transmise. Ces informations étaient collectées dans le but d'expliquer les comportements mis en évidence dans les questions qualitatives.

Une analyse statistique a été réalisée dans le but de caractériser les répondants en fonction des difficultés rencontrées lors de leur installation (pour les nouveaux installés) ou lors de leur parcours de cession (pour les futurs cédants), ainsi que de la difficulté principale. Pour ce faire, nous avons réalisé une classification hiérarchique (ou analyse en clusters) sur chacun des deux sous-échantillons : le souséchantillon des 15 nouveaux installés, et le sous-échantillon des 25 futurs cédants. Cependant, au vu de la faible taille de nos sous-échantillons et des biais inhérents à la sélection des individus (sur-représentation et sous-représentation de certains types d'agriculteur et d'exploitation agricole), les résultats obtenus ont un intérêt limité Ils sont présentés à titre d'information en annexe de notre article (annexes 2 et 3 ) mais sont à considérer avec précaution. Par conséquent, nous nous sommes focalisés sur les analyses descriptives de nos deux sous-échantillons concernant la question des difficultés rencontrées dans leur parcours respectif de transmission et d'installation. Nous terminons par une analyse comparative quantitative et qualitative afin de déterminer les préoccupations à la fois spécifiques et communes aux futurs cédants et nouveaux installés.

\section{Résultats concernant les nouveaux installés}

Le tableau 1 présente les statistiques descriptives des 15 nouveaux installés rencontrés. Ceux-ci ont en moyenne 32 ans et 
Tableau 1. Statistiques descriptives des 15 nouveaux installés enquêtés

\begin{tabular}{lccrr}
\hline Variables & Moyenne & Écart-type & Minimum & Maximum \\
\hline Âge & 32,5 & 5,2 & 25 & 42 \\
Temps de travail hebdomadaire (heures) & 60,7 & 9,2 & 45 & 80 \\
Valeur de l'exploitation reprise (euros) & 245607 & 171185 & 67000 & 641000 \\
SAU (hectares) & 63,8 & 55,6 & 2 & 170 \\
SAU en propriété (hectares) & 8,9 & 11,7 & 0 & 40 \\
SAU en fermage (hectares) & 51,1 & 50,5 & 0 & 170 \\
\hline & & & & \\
\end{tabular}

demi, les deux tiers d'entre eux ayant plus de 30 ans et le plus âgé ayant 42 ans. Un tiers d'entre eux ont un diplôme universitaire. Leur temps de travail hebdomadaire moyen est de 60,7 heures, compris dans une tranche allant de 45 à 80 heures, avec presque les deux tiers travaillant plus de 60 heures par semaine. Un seul a une activité extérieure à l'exploitation. L'installation s'est faite en cadre familial pour 7 nouveaux installés sur 15 et hors cadre familial pour les 8 autres. En moyenne, les répondants se sont installés depuis 45 mois, avec un tiers depuis moins de 30 mois et un tiers depuis plus de 60 mois.

Les nouveaux installés interrogés exploitent une SAU moyenne de 63,8 hectares dont 8,9 hectares en propriété. La plus petite exploitation de l'échantillon a une SAU de 2 hectares et la plus grande une SAU de 170 hectares. La valeur moyenne de l'exploitation reprise était de 245607 euros, avec là aussi une forte variation dans l'échantillon, puisque les valeurs sont comprises entre 67000 euros et 641000 euros. Un seul répondant n'a pas réalisé son parcours dans le cadre d'une installation avec DJA. Les deux tiers des 15 nouveaux installés sont en EARL ou GAEC (dont 7 en EARL), et pour un tiers en entreprise individuelle. Un tiers est sur une exploitation de granivores et les autres ont des orientations productives variées. Un tiers exploite en mode biologique.

Les aspects les plus importants recherchés par les nouveaux installés dans leur choix de l'exploitation à reprendre étaient l'orientation productive de l'exploitation (pour 8 des répondants), une bonne structuration du foncier (pour 6 des répondants) et une bonne rentabilité économique de l'exploitation (pour 6 des répondants).

Le tableau 2 présente les difficultés rencontrées par les nouveaux installés lors de leur installation (les difficultés sont celles qui étaient proposées aux répondants et ne sont pas exclusives), et le tableau 3 présente les difficultés considérées comme principales par les nouveaux installés (il s'agit des mêmes difficultés mais elles sont cette fois exclusives). Selon le tableau 2, la part des nouveaux installés est la plus importante pour la difficulté relative aux contrôles administratifs (type CDOA). Plus précisément, 7 répondants considèrent que les contrôles administratifs étaient une difficulté (parmi d'autres) lors de leur parcours d'installation. La deuxième difficulté regroupant le plus de répondants (4 nouveaux installés) concerne la lourdeur administrative (en particulier les délais), et la troisième difficulté ( 3 des répondants) a trait au financement des capitaux d'exploitation. Ce sont donc les contrôles et les contraintes administratives qui ressortent surtout (11 répondants en cumulé).

Ceci est confirmé par le tableau 3 qui montre la part des nouveaux installés enquêtés selon la difficulté qu'ils considèrent comme principale. La difficulté majeure qui regroupe le plus de répondants (3) concerne les contrôles administratifs, 
RECHERCHE

Romain GATÉ, Laure LATRUFFE

Tableau 2. Part des nouveaux installés enquêtés en fonction des difficultés rencontrées lors du parcours à l'installation

\begin{tabular}{lcc}
\hline Difficultés & Effectifs & Part des nouveaux installés \\
\hline Autre difficulté & 8 & $53 \%$ \\
Les contrôles administratifs & 7 & $47 \%$ \\
La lourdeur administrative & 4 & $27 \%$ \\
Le financement des capitaux d'exploitation & 3 & $20 \%$ \\
L'acceptation du projet par le voisinage & 2 & $13 \%$ \\
Le manque de suivi et de conseil de la Chambre d'agriculture & 2 & $13 \%$ \\
La négociation avec le cédant & 2 & $13 \%$ \\
La durée de création de l'activité & 2 & $13 \%$ \\
Le choix contraint de l'exploitation & 2 & $13 \%$ \\
La mise en concurrence avec plusieurs repreneurs & 1 & $7 \%$ \\
Le morcellement du foncier & 1 & $7 \%$ \\
La recherche du foncier & 1 & $7 \%$ \\
Le financement du foncier & 1 & $7 \%$ \\
L'accord des multiples propriétaires fonciers & 0 & $0 \%$ \\
La durée de la transmission & 0 & $0 \%$ \\
\hline
\end{tabular}

Note : seules ces difficultés étaient proposées aux répondants ; elles sont non exclusives.

Source : les auteurs.

Tableau 3. Part des nouveaux installés enquêtés en fonction de la principale difficulté rencontrée lors du parcours à l'installation

\begin{tabular}{lcc}
\hline Difficulté principale & Effectifs & Part des nouveaux installés \\
\hline Les contrôles administratifs & 3 & $20 \%$ \\
Autre difficulté principale & 3 & $20 \%$ \\
Le financement des capitaux d'exploitation & 2 & $13 \%$ \\
La lourdeur administrative & 2 & $13 \%$ \\
L'acceptation du projet par le voisinage & 2 & $13 \%$ \\
Aucune difficulté principale & 2 & $13 \%$ \\
La mise en concurrence avec plusieurs repreneurs & 1 & $7 \%$ \\
\hline
\end{tabular}

Note : les difficultés du tableau 2 étaient proposées aux répondants, mais sont ici exclusives.

Les difficultés du tableau 2 qui n'apparaissent pas dans le tableau 3 sont celles qui n'ont pas été choisies par les répondants comme difficulté principale ( $0 \%$ de répondants).

Source : les auteurs.

devant la lourdeur administrative (pour 2 des interrogés), le financement des capitaux d'exploitation (pour 2 des interrogés) et l'acceptation du projet par le voisinage (pour 2 autres nouveaux installés).

À noter que, dans les deux tableaux, la part de répondants la plus importante concerne en fait les difficultés « autres» que celles proposées dans la liste. Cela montre que nos hypothèses de base tirées de la littérature sont incomplètes. Cette catégorie regroupe des difficultés variées, comme le manque de certains conseils spécifiques, l'absence de maîtrise des garanties et des cautions bancaires, la méconnaissance de certaines subventions, la recherche de clientèle, etc. Ces difficultés reflètent notamment des problématiques 
liées à des cas particuliers d'installation. L'analyse qualitative des entretiens ne permet pas de les généraliser.

\section{Résultats concernant les futurs cédants}

Le tableau 4 présente les statistiques descriptives des 25 futurs cédants rencontrés. Ceux-ci sont âgés de 56,8 ans en moyenne et cela fait 32 ans en moyenne qu'ils sont chef d'exploitation ou associé exploitant principal dans le cas des formes sociétaires, pour une tranche comprise entre 15 et 40 ans. Aucun n'a un diplôme universitaire. La majorité des futurs cédants n'a pas d'activité en dehors de leur exploitation. Seuls 4 chefs d'exploitation ont une activité salariée hors exploitation et y consacrent en moyenne environ $46,5 \%$ de leur temps de travail hebdomadaire.

Les futurs cédants enquêtés exploitent une SAU moyenne de 86 hectares dont 47,5 hectares sont en fermage en moyenne. Là encore on note une large variation de taille dans l'échantillon, avec une SAU minimale de 38 hectares et une SAU maximale de 230 hectares. Les exploitants sont en EARL et GAEC pour les deux tiers de l'échantillon (notamment EARL pour 10 des enquêtés), et en exploitation individuelle pour 5 d'entre eux. L'exploitation est spécialisée en bovins-lait pour 9 enquêtés, et en cultures-élevage pour 7 futurs cédants. Elle est en agriculture biologique pour 3 des enquêtés.

Tous les futurs cédants interrogés envisagent de transmettre leur outil de travail, en totalité pour 20 d'entre eux et en plusieurs étapes pour 5 d'entre eux. Un futur cédant seulement parmi les interrogés n'a pas encore envisagé la date approximative de sa transmission. Pour la moitié des enquêtés, le successeur est connu et principalement issu du cadre familial. Les trois quarts des futurs cédants interrogés veulent conserver des terres en propriété, à raison de 24,5 hectares en moyenne. Enfin, on peut noter que la réforme de la PAC de 2013 ne les a pas fait modifier leur date de transmission.

En ce qui concerne les stratégies d'investissement avant transmission, 14 des futurs cédants interrogés souhaitent augmenter leurs investissements, et 10 souhaitent seulement maintenir leurs dépenses d'investissement dans leur outil de travail. Seul un répondant a indiqué qu'il souhaitait désinvestir. Parmi les futurs cédants qui souhaitent investir plus, 12 le font pour préparer le développement futur de leur exploitation. Parmi les futurs cédants qui maintiennent leurs dépenses d'investissement, 6 le font pour respecter des contraintes de mise aux normes ou pour renouveler des capitaux d'exploitation. Sur l'ensemble de leur stratégie de préparation de l'exploitation avant la transmission, on note également que 17 exploitants ne changeraient pas leur choix en termes d'investissement si les subventions de la PAC s'arrêtaient en 2020.

Le tableau 5 présente les difficultés rencontrées par les futurs cédants lors de leur démarche de transmission (les difficultés sont celles qui étaient proposées aux

Tableau 4. Statistiques descriptives des 25 futurs cédants enquêtés

\begin{tabular}{lcccc}
\hline Variables & Moyenne & Écart-type & Minimum & Maximum \\
\hline Âge & 56,8 & 3,0 & 50 & 61 \\
Durée avant la transmission (mois) & 48,7 & 33,3 & 3 & 120 \\
SAU (hectares) & 86,0 & 49,5 & 38 & 230 \\
SAU en propriété (hectares) & 28,2 & 24,7 & 5 & 100 \\
SAU en fermage (hectares) & 47,5 & 38,3 & 4 & 174 \\
\hline
\end{tabular}


RECHERCHE

Romain GATÉ, Laure LATRUFFE

Tableau 5. Part des futurs cédants enquêtés en fonction des difficultés rencontrées lors de la transmission

\begin{tabular}{lcc}
\hline Difficultés & Effectifs & Part des futurs cédants \\
\hline La recherche d'un repreneur & 8 & $32 \%$ \\
La lourdeur administrative & 7 & $28 \%$ \\
Autre difficulté & 5 & $20 \%$ \\
L'entente avec les propriétaires fonciers & 4 & $16 \%$ \\
Le morcellement du foncier & 3 & $12 \%$ \\
Le montage juridique et fiscal de cession & 3 & $12 \%$ \\
Le niveau de la retraite espérée & 3 & $12 \%$ \\
Aucune difficulté & 3 & $12 \%$ \\
L'entente au sein de la famille & 1 & $4 \%$ \\
L'entente entre les associés & 1 & $4 \%$ \\
L'entente avec la profession & 1 & $4 \%$ \\
L'entente avec le repreneur & 1 & $4 \%$ \\
Le statut juridique de l'exploitation & 1 & $4 \%$ \\
La durée de la transmission & 1 & $4 \%$ \\
L'entente avec le voisinage & 0 & $0 \%$ \\
Les dates de départ et d'installation qui ne coïncident pas & 0 & $0 \%$ \\
\hline
\end{tabular}

Note : seules ces difficultés étaient proposées aux répondants ; elles sont non exclusives.

Source : les auteurs.

Tableau 6. Part des futurs cédants enquêtés en fonction de la principale difficulté lors de la transmission

\begin{tabular}{lcc}
\hline Difficulté principale & Effectifs & Part des futurs cédants \\
\hline La recherche d'un repreneur & 5 & $20 \%$ \\
Aucune difficulté principale & 4 & $16 \%$ \\
L'entente avec les propriétaires fonciers & 3 & $12 \%$ \\
La lourdeur administrative & 3 & $12 \%$ \\
Autre difficulté principale & 3 & $12 \%$ \\
Le morcellement du foncier & 2 & $8 \%$ \\
Le montage juridique et fiscal de cession & 2 & $8 \%$ \\
Le niveau de la retraite espérée & 1 & $4 \%$ \\
L'entente au sein de la famille & 1 & $4 \%$ \\
\hline
\end{tabular}

Note : les difficultés du tableau 5 étaient proposées aux répondants, mais sont ici exclusives. Les difficultés du tableau 5 qui n'apparaissent pas dans le tableau 6 sont celles qui n'ont pas été choisies par les répondants comme difficulté principale (0\% de répondants).

Source : les auteurs.

répondants et ne sont pas exclusives), et le tableau 6 présente les difficultés considérées comme principales par les futurs cédants (il s'agit des mêmes difficultés mais elles sont cette fois exclusives). D'après le tableau 5, 8 futurs cédants considèrent la recherche d'un repreneur comme une difficulté importante. La lourdeur administrative ainsi que les problématiques foncières (entente avec les propriétaires et morcellement) font également partie des préoccupations des futurs cédants ( 7 interrogés pour chacune des deux difficultés). Le troisième type de difficulté concerne 
d'autres considérations que nous n'avions pas envisagées dans notre liste prédéfinie. Cependant, parmi les 5 futurs cédants qui ont évoqué une autre difficulté, 3 insistent sur la nécessité d'avoir un repreneur qui correspond à un profil idéal afin de pérenniser l'exploitation et les orientations technico-économiques choisies par le cédant.

Le tableau 6 présente les difficultés principales rencontrées par les futurs cédants lors de leur démarche de transmission (les difficultés sont celles qui étaient proposées aux répondants et sont exclusives). Les chiffres montrent que les futurs cédants interrogés indiquent, dans la liste qui leur était proposée, que la difficulté principale concerne surtout la recherche du repreneur (pour 5 des interrogés), devant la lourdeur administrative et l'entente avec les propriétaires fonciers (pour 3 futurs cédants dans les deux cas). Ces observations confirment les enseignements tirés du tableau 5 : trouver son successeur idéal, et faire face aux contraintes administratives et foncières sont les principaux obstacles à franchir pour les futurs cédants de notre échantillon. Il apparaît également que 4 futurs cédants ne rencontrent aucune difficulté principale. Alors que deux d'entre eux n'observent aucune difficulté particulière, deux considèrent la recherche du repreneur et la lourdeur administrative comme des freins mineurs. Il n'existe donc pas toujours une difficulté déterminante, mais le cumul de difficultés mineures peut représenter un frein à la transmission.

\section{Analyse comparative entre les futurs cédants et les nouveaux installés}

Le tableau 7 permet d'identifier les différences et les similitudes dans les difficultés que rencontrent les futurs cédants et les nouveaux installés lors de la transmission d'une exploitation. Ce tableau représente les difficultés proposées aux futurs cédants et aux nouveaux installés lors de l'enquête, ainsi que les difficultés spécifiques qui leur étaient proposées. Les difficultés communes et les difficultés spécifiques sont rangées par ordre décroissant en rapport avec les tableaux 2 et 5 précédents. Les difficultés ayant été sélectionnées par 3 individus ou plus apparaissent en gras.

Les difficultés qui avaient été proposées aux futurs cédants et aux nouveaux installés regroupent les relations avec les propriétaires fonciers et le voisinage, l'entente cédant-repreneur lors de la négociation ainsi que les questions liées à la structure foncière, à la durée de la transmission et à la lourdeur administrative. Parmi les difficultés communes, la lourdeur administrative est la difficulté la plus partagée par les deux parties, avec 11 répondants cumulés qui l'ont mentionnée. Les problèmes liés au foncier apparaissent en deuxième position, avec 4 répondants (seulement des futurs cédants) trouvant difficiles les relations avec les propriétaires fonciers, et 4 répondants (3 futurs cédants et 1 nouvel installé) identifiant le problème du morcellement du foncier. La relation cédant-repreneur vient ensuite, relevée par 3 interrogés (1 futur cédant et 2 nouveaux installés). La durée de la transmission n'a été relevée que par une personne, plus spécifiquement un cédant. Ces chiffres indiquent donc une lourdeur administrative effective pour les deux parties, et des problèmes liés au foncier perçus principalement par les cédants.

Quant aux difficultés spécifiques, si on observe que la recherche du repreneur est une préoccupation majeure (8 futurs cédants), les difficultés spécifiques principalement identifiées par les deux parties ont également trait aux freins administratifs : montage juridique et fiscal pour 3 futurs cédants, et contrôles administratifs pour 7 nouveaux installés. Ces contrôles sont liés notamment au Plan de développement économique $(\mathrm{PDE})^{4}$ que doivent respecter

4. Un changement du PDE entraîne une pénalité financière. 
RECHERCHE

Romain GATÉ, Laure LATRUFFE

Tableau 7. Comparaison des difficultés rencontrées par les futurs cédants et nouveaux installés lors de la transmission

\begin{tabular}{|c|c|c|c|c|}
\hline Difficultés & Futurs cédants & Effectifs & Nouveaux installés & Effectifs \\
\hline \multirow{6}{*}{ Communes } & La lourdeur administrative & 7 & La lourdeur administrative & 4 \\
\hline & $\begin{array}{l}\text { L'entente avec les propriétaires } \\
\text { fonciers }\end{array}$ & 4 & $\begin{array}{l}\text { L'acceptation du projet } \\
\text { par le voisinage }\end{array}$ & 2 \\
\hline & Le morcellement du foncier & 3 & La négociation avec le cédant & 2 \\
\hline & L'entente avec le repreneur & 1 & Le morcellement du foncier & 1 \\
\hline & La durée de la transmission & 1 & $\begin{array}{l}\text { L'accord des multiples propriétaires } \\
\text { fonciers }\end{array}$ & 0 \\
\hline & L'entente avec le voisinage & 0 & La durée de la transmission & 0 \\
\hline \multirow{10}{*}{ Spécifiques } & La recherche d'un repreneur & 8 & Autre difficulté & 8 \\
\hline & Autre difficulté & 5 & Les contrôles administratifs & 7 \\
\hline & $\begin{array}{l}\text { Le montage juridique et fiscal } \\
\text { de cession }\end{array}$ & 3 & $\begin{array}{l}\text { Le financement des capitaux } \\
\text { d'exploitation }\end{array}$ & 3 \\
\hline & Le niveau de la retraite espérée & 3 & $\begin{array}{l}\text { Le manque de suivi et de conseil } \\
\text { de la Chambre d'agriculture }\end{array}$ & 2 \\
\hline & Aucune difficulté & 3 & La durée de création de l'activité & 2 \\
\hline & L'entente au sein de la famille & 1 & Le choix contraint de l'exploitation & 2 \\
\hline & L'entente entre les associés & 1 & $\begin{array}{l}\text { La mise en concurrence avec } \\
\text { plusieurs repreneurs }\end{array}$ & 1 \\
\hline & L'entente avec la profession & 1 & La recherche du foncier & 1 \\
\hline & Le statut juridique de l'exploitation & 1 & Le financement du foncier & 1 \\
\hline & $\begin{array}{l}\text { Les dates de départ et d'installation } \\
\text { qui ne coïncident pas }\end{array}$ & 0 & & \\
\hline
\end{tabular}

Note : il s'agit des difficultés présentées dans les tableaux 2 et 5.

Source : les auteurs.

les jeunes agriculteurs recevant des aides publiques mais aussi à la Direction générale de la concurrence, de la consommation et de la répression des fraudes (DGCCRF) pour ceux qui créent une nouvelle activité incluant la transformation de produit. En troisième lieu, on remarque des difficultés spécifiques ayant trait aux aspects financiers : 3 futurs cédants sont mécontents du niveau de retraite espéré et 3 nouveaux installés indiquent que le financement des capitaux est problématique.

En comparant les difficultés principales vécues par les deux parties (tableau 8), on retrouve ainsi cette opposition entre des futurs cédants inquiets quant à leur capacité de trouver un successeur idéal pour leur exploitation et de l'autre des nouveaux installés freinés par les contrôles administratifs. Cependant, il faut noter que 4 cédants sur 25 ne considèrent pas de frein principal dans leur démarche de transmission tandis que 3 nouveaux installés sur 15 font ressortir des difficultés principales que nous n'avions pas prédéterminées.

\section{Apports qualitatifs}

Tous les nouveaux installés enquêtés avaient bénéficié d'aides publiques pour leur reprise ou création d'activité, alors qu'au niveau national sur la période 20032010 parmi les installés de moins de 40 ans qui sont potentiellement éligibles DJA, près de 50-55\% d'entre eux ne remplissaient pas les conditions pour l'obtenir ou ne la demandaient pas (APCA, 2013). Ceci peut expliquer partiellement le poids des freins administratifs dans les difficultés évoquées 
Tableau 8. Comparaison des difficultés principales rencontrées par les futurs cédants et les nouveaux installés lors de la transmission

\begin{tabular}{l|lc|lc}
\hline \multicolumn{1}{c}{ Futurs cédants } & \multicolumn{1}{c}{ Effectifs } & \multicolumn{1}{c}{ Nouveaux installés } & Effectifs \\
\hline \multirow{4}{*}{$\begin{array}{l}\text { Difficultés } \\
\text { principales }\end{array}$} & La recherche d'un repreneur & 5 & Les contrôles administratifs & 3 \\
Aucune difficulté principale & 4 & Autre difficulté principale & 3 \\
L'entente avec les propriétaires & 3 & Le financement des capitaux & 2 \\
fonciers & & d'exploitation & La lourdeur administrative & 2 \\
La lourdeur administrative & 3 & L'acceptation du projet par le voisinage & 2 \\
& Autre difficulté principale & 3 & Aucune difficulté principale & 2 \\
Le morcellement du foncier & 2 & La mise en concurrence avec plusieurs & 1 \\
Le montage juridique et fiscal & 2 & repreneurs & \\
de cession & 1 & & \\
Le niveau de la retraite espérée & 1 & & \\
L'entente au sein de la famille & 1 & &
\end{tabular}

Note : il s'agit des difficultés qui ont été présentées dans les tableaux 3 et 6.

Source : les auteurs.

par les enquêtés. Certains nouveaux installés enquêtés ont insisté sur la question des délais à respecter dans l'obtention de l'autorisation d'exploiter tandis que d'autres ont évoqué la difficulté à « rentrer dans les cases » des contrôleurs. Les autres difficultés évoquées par les nouveaux installés sont spécifiques à leur situation : recherche de clientèle, acceptation difficile du projet d'élevage de porc par le voisinage, manque de conseils de la part d'un technicien caprin, question du partage de la maison d'habitation entre frères et sœurs, etc.

Concernant les futurs cédants, 12 d'entre eux connaissaient leur successeur au moment de l'enquête et 13 n'en avaient toujours pas trouvé. Pourtant, cette incertitude n'empêche pas 6 interrogés sur 13 de poursuivre le développement futur de leur exploitation par des investissements en l'absence de successeur. Les autres préoccupations principales évoquées pendant les entretiens concernent avant tout les volets administratifs et fonciers. Parmi les autres difficultés évoquées, deux agriculteurs en système biologique sur les 3 du souséchantillon attachent de l'importance à ce que le repreneur ait un profil proche du leur afin de garantir le maintien du système en place. Certains futurs cédants en agriculture conventionnelle y accordent la même importance, quelle que soit l'orientation technico-économique de leur exploitation. Deux futurs cédants évoquent le problème de rentabilité de leur exploitation (une en bovins laitiers et l'autre en culture céréalière) qui la rend, de fait, moins attractive pour un repreneur et qui peut alors nécessiter une diversification du système technique de la part du repreneur.

Ces enseignements sont utiles afin de prolonger cette recherche exploratoire par une étude plus approfondie permettant de tester certaines hypothèses. Par exemple, les nouveaux installés hors cadre familal ont-ils plus de difficultés à s'installer contrairement à leurs homologues dans le cadre familial ? Quels sont les freins et menaces qui les distinguent particulièrement des installés en cadre familial lors de la reprise ou création de l'exploitation et peut-on mieux les accompagner ? Cette question nous paraît essentielle au vu du nombre important de nouveaux installés hors cadre familial faisant part d'autres difficultés dans les entretiens ( 7 sur 8 installés hors cadre familial interrogés). Un autre exemple d'analyse plus poussée concerne 
les stratégies d'investissement des futurs cédants. Celles-ci sont-elles liées à la présence ou à l'absence d'un successeur ? La faible taille de notre échantillon ne nous permet pas de conclure de manière robuste sur ce point et nécessiterait un panel plus conséquent. Il serait notamment intéressant de comparer ces stratégies en fonction du statut juridique de l'exploitation, en prenant en compte la facilité d'accès aux prêts, la rentabilité, la capacité de remboursement, ou le turn-over des associés au sein des formes sociétaires.

*

Si ce travail se doit d'être poursuivi par une recherche plus poussée, notamment avec des analyses statistiques sur un échantillon d'agriculteurs plus grand et plus représentatif de la diversité des situations, il nous permet de dégager quelques conclusions intéressantes.

Tout d'abord, on peut souligner un certain dynamisme des transmissions dans la région Bretagne, avec un accompagnement (conseillers, comptables, banquiers, etc.) qui semble satisfaisant pour la plupart des personnes interrogées. Néanmoins, la complexité et la lourdeur administratives ainsi que le volet foncier (structure et entente avec les propriétaires) sont des aspects clefs qui pourraient à l'avenir fragiliser ce dynamisme. Ces aspects, relevés dans la littérature et par les experts, sont également mis en avant par les agriculteurs enquêtés, que ce soit les nouveaux installés ou les futurs cédants. Concernant l'installation de plus en plus prégnante des jeunes agriculteurs hors cadre familial, notre étude exploratoire relève des différences vis-à-vis des nouveaux installés en cadre familial. Les installés hors cadre familial rencontreraient plus fréquemment d'autres difficultés que les autres, en sus des freins couramment relevés dans la littérature.

Ensuite, on observe que certains futurs cédants anticipent leur transmission en adoptant une stratégie d'investissement spécifique, qui éviterait au repreneur d'avoir à mettre en place ces investissements, favoriserait la continuité de l'activité productive et diminuerait ainsi le coût global de reprise. Sur ce point, on peut insister sur la nécessaire anticipation des exploitants concernant la cession de leur exploitation. Celle-ci permet, outre de mettre en place une stratégie d'investissement, également de trouver le repreneur idéal, monter le dossier administratif et financier, gérer les relations avec les parties prenantes (famille, voisinage, propriétaires fonciers, etc.) et ainsi préparer au mieux la pérennité de l'exploitation et la période de retraite du cédant. Ceci est d'autant plus important que le successeur n'est pas identifié. Il est surprenant que la question de la relation cédant-repreneur ait été très peu évoquée par les nouveaux installés interrogés et peu anticipée comme problématique par les futurs cédants interrogés, alors que la littérature et les experts rencontrés ont identifié ce point comme crucial lors de la transmission d'une exploitation agricole.

Différents leviers d'accompagnement peuvent être actionnés afin de modérer les difficultés rencontrées. Par exemple, une sensibilisation systématique dix ans avant l'âge légal de départ en retraite, réalisée par les acteurs privés et publics à destination des agriculteurs sur la démarche de transmission, pourrait permettre d'anticiper les questions administratives, fiscales, juridiques et foncières et permettrait de lever les incertitudes sur l'avenir de leurs outils de production. De plus, le contrat de génération en agriculture proposé et accepté dans la LOA 2014 pourrait être un moyen de favoriser l'installation progressive d'un repreneur tout en permettant au cédant de quitter son activité progressivement en transmettant son savoir-faire et en sécurisant l'avenir de son exploitation.

Enfin, on peut soulever deux points peu abordés dans ce travail mais qui méritent 
des recherches plus poussées. Le premier point concerne l'augmentation des installations dans le cadre de formes sociétaires. Ceci peut impliquer le besoin de capacités de management pour les repreneurs, puisque ceux-ci devront être capables de gérer à la fois l'économique, l'humain et la technique pour maintenir leur exploitation. Le deuxième point concerne le foncier, pour lequel un problème crucial risque de se poser dans les années à venir, puisque les cédants transmettent rarement la totalité de leurs terres en propriété. Les nouveaux installés détiennent de moins en moins leur terre en pleine propriété et seront de plus en plus dépendants de leur(s) propriétaire(s) foncier(s) vis-à-vis de la transmission future de leur terre avec l'exploitation, ce qui peut fragiliser le devenir de l'outil agricole.

Les auteurs remercient la chaire " Entreprises et Économie Agricole » (CréditAgricole en Bretagne et Agrocampus Ouest) pour son soutien financier et matériel lors de cette étude. Nous remercions également les deux relecteurs anonymes pour leurs critiques et remarques constructives ainsi que les commentaires bienvenus de Vincent Chatellier qui ont contribué à l'amélioration de cet article.

\section{RÉFÉRENCES BIBLIOGRAPHIQUES}

Agreste (2014). Recensement agricole 2010. Résultats et données chiffrées. En ligne: http://www.agreste.agriculture.gouv.fr/ recensement-agricole-2010/resultats-donnees-chiffrees/

APCA (2013). Analyses et perspectives. Regards sur les installations d'agriculteurs entre 1997 et 2010. Assemblée permanente des chambres d'agriculture. Février 2013, $n^{\circ}$ 1303. En ligne: http://www.installagri. net/etudes/Etude2013.pdf

Barthélémy D. (1997). La valeur de l'entreprise agricole. INRA Sciences sociales, vol. 10, $\mathrm{n}^{\circ} 1,4 \mathrm{p}$.

Barthez A., Charbonnier E. (2003). Conseiller la transmission en agriculture. Enquête auprès de 22 conseillers en Adasea. Trame, 66 p.

Bennedsen M., Nielsen K.M., PerezGonzalez F., Wolfenzen D. (2007). Inside the family firm: the role of families in succession decisions and performance. Quarterly Journal of Economics, vol. 122, pp. 647-691.

Bessière C. (2006). Maintenir une entreprise familiale : enquête sur les exploitations viticoles de la région délimitée Cognac, thèse de doctorat en sociologie, sous la direction d'Olivier Schwartz, Paris, Université Paris V, $602 \mathrm{p}$
Bessière C. (2008). Se marier pour aller jusqu'au bout ensemble? Ruptures conjugales et transmission des exploitations agricoles dans la lignée. Review of Agricultural and Environmental Studies-Revue d'Études en Agriculture et Environnement, vol. 88, $\mathrm{n}^{\circ} 3$, pp. 47-70.

Boinon J.-P. (2011). Les politiques foncières agricoles en France depuis 1945. Économie et Statistique, vol. 444, $\mathrm{n}^{\circ}$ 1, pp. 19-37.

Cadieux L., Gratton P., St-Jean É. (2015). La carrière repreneuriale: contexte et défis. Revue de l'entrepreneuriat, vol. $13, \mathrm{n}^{\circ} 1$, pp. 35-50.

Calus M., Van Huylenbroeck G., Van Lierde D. (2008). The relationship between farm succession and farm assets on Belgian farms. Sociologia ruralis, vol. 48, $\mathrm{n}^{\circ} 1$, pp. 38-56.

Chambres d'agriculture de Bretagne (2014). Note transmission, la mise en auvre des Points Accueil Transmission, groupe de travail du 28 avril 2014. Conclusions du comité de pilotage du 30 janvier 2014.

David J. (1988). Les formes contemporaines de la transmission des exploitations agricoles. Études rurales, pp. 71-83. 
RECHERCHE

Gault J., Marty S., Menard J.-N.,Pringault J.-M. (2013a). Évaluation des mesures prises dans le cadre de la Loi d'orientation agricole de 2006 pour faciliter la transmission des exploitations agricoles et le financement des facteurs de production par des capitaux extérieurs. Ministère de l'Agriculture, de l'Agroalimentaire et de la Forêt. Tome 1, $42 \mathrm{p}$.

Gault J., Marty S., Menard J.-N.,Pringault J.-M. (2013b). Évaluation des mesures prises dans le cadre de la Loi d'orientation agricole de 2006 pour faciliter la transmission des exploitations agricoles et le financement des facteurs de production par des capitaux extérieurs. Ministère de l'Agriculture, de l'Agroalimentaire et de la Forêt. Tome 2, $197 \mathrm{p}$.

Gillet M. (1999). Analyse sociologique des transmissions dites hors cadre familial des exploitations agricoles. Économie rurale, vol. $253, \mathrm{n}^{\circ} 1$, pp. 87-90.

Hair J., Anderson R., Tatham R., Black W. (1998). Multivariate Data Analysis. Cinquième édition. Prentice-Hall International, New Jersey, États-Unis, 730 p.

Jacques-Jouvenot D. (1997). Choix du successeur et transmission patrimoniale. Paris, L'Harmattan, coll. «Logiques sociales », $239 \mathrm{p}$.

Jacques-Jouvenot D., Schepens F. (2007). Transmettre et reprendre une entreprise: de l'Homo œconomicus à l'Homo memor. Revue du MAUSS, vol. 29, n $^{\circ}$ 1, pp. 377-391

Jacques-Jouvenot D. (2012). Se séparer sans rompre ou l'art de transmettre. In « Biennale internationale de l'éducation, de la formation et des pratiques professionnelles», Paris, $9 \mathrm{p}$.

Jacques-Jouvenot D. (2014a). Le paradoxe de la transmission du métier: le cas des éleveurs. Sociologies. En ligne : http://sociologies.revues.org/4566

Jacques-Jouvenot D. (2014b). Une hypothèse inattendue à propos du suicide des éleveurs : leur rapport aux savoirs professionnels. Études rurales, vol. 193, n 1, pp. 45-60.

Jeunes Agriculteurs (2014). Les aides et les financements. En ligne: http://www. jeunes-agriculteurs.fr/devenir-agriculteur/ reprise-creation-entreprise-agriculture/ item/17-aides-financements

La France Agricole (2014a). Se préparer à transmettre ses biens et son exploitation. Hors-série Transmission. Paris, France Agricole, février, $90 \mathrm{p}$.

La France Agricole (2014b). Conférence au Salon de l'Agriculture sur la réussite de la transmission d'exploitation agricole. En ligne : http://www.lafranceagricole.fr/videoet-photo-agricole/actualites-evenements/ sia-2014-une-conference-pour-reussir-satransmission-d-exploitation-partie-1-85253. html\#player.

Latruffe L., Le Mouël C. (2009). Capitalization of government support in agricultural land prices: What do we know? Journal of Economic Surveys, vol. 23, n 4, pp. 659-691.

Levinson H. (1971). Conflicts that plague family businesses. Harvard Business Review, vol. $49, n^{\circ} 2$, pp. 90-98.

Lobley M., Baker J., Whitehead I. (2010). Farm succession and retirement: some international comparisons. Journal of Agriculture, Food Systems, and Community Development, vol. 1, $\mathrm{n}^{\circ}$ 1, p. 49-64.

Mishra A.K., El-Osta H.S. (2008). Effect of agricultural policy on succession decisions of farm households. Review of Economics of the Household, vol. 6, $\mathrm{n}^{\circ}$ 3, p. 285-307.

Pelc A. (2014). Tableau de bord de la population des nouveaux exploitants agricoles en 2012. Direction des études des répertoires et des statistiques. En ligne : http://www.msa. $\mathrm{fr} / \mathrm{lfr} /$ etudes-statistiques/tdb.

Safer-SSP-Terres d'Europe-Scafr-INRA (2014). L'évolution des prix. Prix des terres et prés. En ligne : http://www.le-prix-des-terres.fr/.

Souma T., Kiminami L. (2011). Knowledge management in agricultural succession. (In Japanese. With English summary.). Studies in Regional Science, vol. 41, n 4, pp. 943-955.

Verzele F., Crijns H. (2001). Les freins à la création d'entreprises. $28 \mathrm{p}$.

Wheeler S., Bjornlund H., Zuo A., Edwards J. (2012). Handing down the farm? The increasing uncertainty of irrigated farm succession in Australia. Journal of Rural Studies, vol. 28, n 3, pp. 266-275. 


\section{ANNEXES}

Annexe 1. Montant de la Dotation Jeunes Agriculteurs (DJA)

\begin{tabular}{|l|c|c|c|}
\hline Zones & Montant minimum & Montant moyen & Montant maximum \\
\hline Plaine & $8000 €$ & $12650 €$ & $17300 €$ \\
\hline Défavorisée & $10300 €$ & $16350 €$ & $22400 €$ \\
\hline Montagne et Outre-Mer & $16500 €$ & $26200 €$ & $35900 €$ \\
\hline
\end{tabular}

Source : Jeunes Agriculteurs (2014).

\section{Annexe 2. Classification hiérarchique exploratoire de 11 nouveaux installés enquêtés selon les difficultés rencontrées lors de leur parcours à l'installation}

Une classification hiérarchique (Hair et al., 1998) a été réalisée à titre exploratoire afin de regrouper les nouveaux installés enquêtés en fonction des difficultés rencontrées à l'installation, que ce soit les difficultés de façon non exclusive, mais également la difficulté principale dans cette liste de difficultés. D'après le dendrogramme et les indices de Duda-Hart (indice Je(2)/Je(1) le plus élevé possible associé à un pseudo- $\mathrm{t}^{2}$ le plus faible possible) obtenus pour cette classification hiérarchique des 15 nouveaux installés (voir ci-dessous), deux groupes sont statistiquement appropriés, mais un des deux groupes compte 14 individus alors que l'autre groupe n'en compte qu'un seul. Nous avons donc retiré l'individu isolé et refait une classification sur les 14 nouveaux installés restants. Les résultats indiquent que trois groupes sont appropriés, mais un groupe comporte 11 individus et chacun des deux autres groupes compte un faible nombre d'individus (1 et 2 individus respectivement) rendant difficile l'interprétation des résultats. Nous avons de nouveau retiré ces individus isolés pour réaliser une classification sur les 11 individus restants. Suite à cela, nous avons sélectionné deux groupes (avec 8 individus et 3 individus respectivement) car même si le rapport Je(2)/ $\mathrm{Je}(1)$ n'est pas le plus élevé, le pseudo- $\mathrm{t}^{2}$ associé est l'un des plus faibles.

En ce qui concerne les variables utilisées pour créer les groupes, seule la difficulté majeure discrimine statistiquement les deux groupes. Le groupe avec 3 individus considère que la difficulté majeure à l'installation concerne les contrôles administratifs, alors que le groupe avec 8 individus considère des difficultés majeures variées. En ce qui concerne la caractérisation des profils des deux groupes, une différence statistiquement significative n'est observée que pour l'opinion concernant les dispositifs d'accompagnement à l'installation. Le groupe réunissant les 8 individus estime ces dispositifs comme suffisants, alors que le groupe réunissant les 3 individus, qui considèrent tous les contrôles administratifs comme la difficulté majeure à l'installation, estime que les dispositifs d'accompagnement ne sont pas suffisants. 


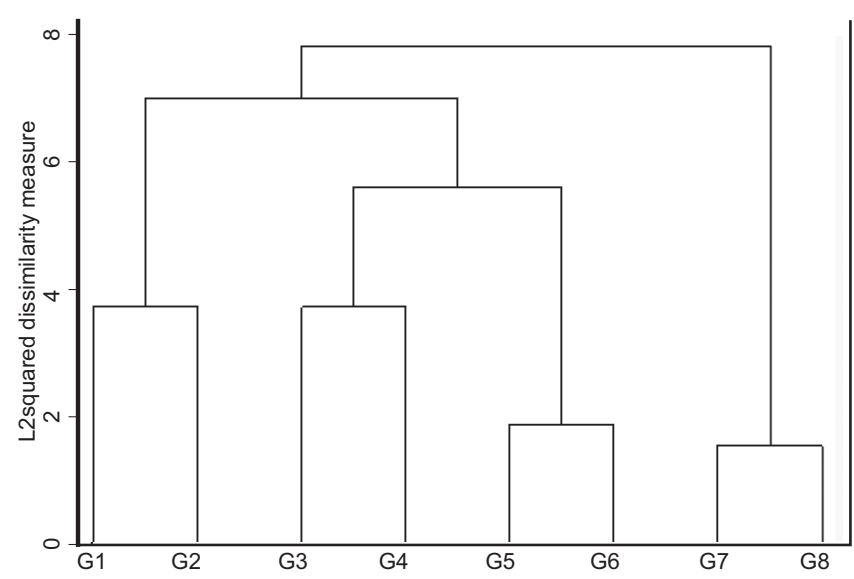

Source : les auteurs.

Indices de Duda-Hart selon le nombre de groupes

\begin{tabular}{|l|c|c|}
\hline Nombre de groupes & Je(2)/Je(1) & Pseudo-t $^{2}$ \\
\hline 1 & 0,7575 & 2,88 \\
\hline 2 & 0,6809 & 2,81 \\
\hline 3 & 0,5000 & 3,00 \\
\hline 6 & 0,0000 & - \\
\hline 7 & 0,3750 & 1,67 \\
\hline 8 & 0,0000 & - \\
\hline
\end{tabular}

Source : les auteurs.

\section{Annexe 3. Classification hiérarchique des 25 futurs cédants enquêtés selon les difficultés rencontrées lors de leur démarche de transmission}

Comme pour les nouveaux installés, la classification hiérarchique pour les futurs cédants rencontrés a été réalisée sur les difficultés sélectionnées par ceux-ci ainsi que sur la difficulté qu'ils ont identifiée comme étant la principale. Le dendrogramme ainsi que les indices de Duda-Hart (voir ci-dessous) indiquent une classification en deux groupes (la valeur de $\mathrm{Je}(2) / \mathrm{Je}(1)$ est une des plus élevées et le pseudo- $\mathrm{t}^{2}$ est parmi les plus faibles). Les deux groupes comportent respectivement 19 individus et 6 individus.

Ces groupes sont statistiquement différents entre eux pour deux variables utilisées pour réaliser la classification : la difficulté liée à la recherche d'un repreneur, et la difficulté principale qui reflète également le problème de la recherche d'un repreneur. C'est cette recherche qui est le plus problématique pour la majorité du groupe de 6 individus, alors que les 19 individus de l'autre groupe sont répartis selon quelques difficultés : morcellement $\mathrm{du}$ foncier, mauvaise entente avec les propriétaires fonciers, montage juridique et fiscal de cession, et lourdeur administrative. En ce qui concerne le profil des deux groupes, la comparaison statistique des caractéristiques de chacun des groupes montre que ceux-ci sont statistiquement différents entre eux en ce qui concerne la présence d'un successeur. Plus précisément, le groupe des 6 individus, qui considère la recherche d'un repreneur comme la principale difficulté, n'a pas de successeur. En revanche, les futurs cédants de l'autre groupe connaissent leur successeur pour 13 d'entre eux dont 10 pour qui le repreneur est un membre de la famille. 
Difficultés rencontrées lors de la transmission d'exploitations agricoles

Dendrogramme à dix groupes

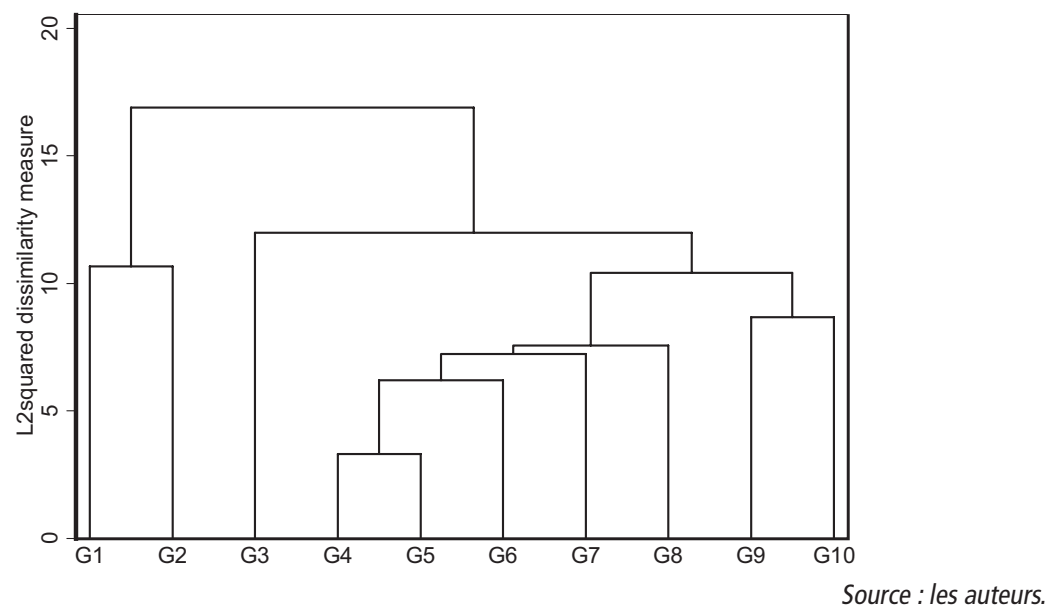

Indices de Duda-Hart selon le nombre de groupes

\begin{tabular}{|l|c|c|}
\hline Nombre de groupes & Je(2)/Je(1) & Pseudo-t ${ }^{2}$ \\
\hline 1 & 0,8278 & 4,78 \\
\hline 2 & 0,8199 & 3,74 \\
\hline 3 & 0,2727 & 10,67 \\
\hline 4 & 0,7991 & 3,52 \\
\hline 5 & 0,2778 & 7,80 \\
\hline 6 & 0,7431 & 3,11 \\
\hline 7 & 0,6543 & 3,70 \\
\hline 8 & 0,4364 & 5,17 \\
\hline 9 & 0,3125 & 6,60 \\
\hline 10 & 0,3333 & 6,00 \\
\hline 11 & 0,0000 & - \\
\hline 14 & 0,0000 & - \\
\hline
\end{tabular}

Source : les auteurs. 